\title{
Exposed cervical dentin and dentin hypersensitivity summary of the discussion and recommendations
}

\author{
Cor van Loveren
}

Received: 23 October 2012 / Accepted: 23 November 2012 / Published online: 9 December 2012

(C) Springer-Verlag Berlin Heidelberg 2012

Keywords Exposed cervical dentin · Dentin

hypersensitivity $\cdot$ Oral health-related quality of life

\section{General discussion}

First, the question was addressed whether exposed cervical dentin deserves attention irrespective if the individual suffers from dentin hypersensitivity. All presentations had, however, discussed dentin hypersensitivity implicitly suggesting that symptom-free exposed cervical dentin does not warrant special treatment although these areas are at risk for dentin hypersensitivity but also for cervical caries. These risks were recognized by the forum, and in the final management scheme, it is advised that exposed cervical dentin is further investigated for hypersensitivity and that in absence of dentin hypersensitivity the patient is advised on the possible future presentation of it [1]. The forum, however, recognized the risk of creating patients, which should not be encouraged.

All presentations adhered to a similar definition of dentin hypersensitivity: Dentin hypersensitivity is characterized by distinctive short sharp pain arising from exposed cervical dentin in response to various external stimuli that are typically thermal, evaporative, tactile, electrical, osmotic, or chemical and which cannot be ascribed to any other form of dental pathology defect or disease [2]. Several aspects of this definition were discussed. It was questioned why the definition mention hypersensitivity. There is no evidence that there are any biological changes making the tooth more sensitive than other teeth neither that pulp pathology is

C. van Loveren $(\square)$

Department of Preventive Dentistry,

Academic Centre for Dentistry Amsterdam,

University of Amsterdam and VU University,

Gustav Mahlerlaan 3004,

1081 LA Amsterdam, The Netherlands

e-mail: c.van.loveren@acta.nl involved [3]. The difference between sensitive and nonsensitive dentin relates primarily to the number and size of patent dentin tubules [3]. Perhaps it would be better to use the term dentin sensitivity. Coronal exposed dentin may also present (hyper)sensitivity. Probably, the mechanisms are the same as for cervical dentin (hyper)sensitivity and differences in the degree of sensitivity are related to the number and shape of the dentinal tubules [3].

A third point of discussion in relation to the definition is the cause of exposure of the dentin. The forum agreed on the role of gingival recession and that the exposure should be non-caries related. The exposure could be caused by either abrasion, erosion, attrition, or abfraction, or clinically more relevant a combination of these. Finally, it was remarked that not the dentin but pulp is sensitive. In spite of all these comments on the definition, the forum suggested for clarity reason to stay in line with the common use and to continue to use the term dentin hypersensitivity.

\section{Epidemiology}

It became clear that there is only limited epidemiological data available on the prevalence and the natural course of dentin hypersensitivity [4]. The reported prevalence data varies enormously depending on the selected population. It is clear that it is prevalent after periodontal treatment but the larger part of dentin hypersensitivity after periodontal treatment will faint out in 2 to 3 weeks [5]. It was estimated that 2 to $6 \%$ of periodontitis patients develop continuous dentin hypersensitivity, but there are no studies substantiating these figures. The suggestion to conclude each scaling and root planing procedure routinely by a preventive (desensitizing) treatment, e.g., fluoride application or prescription of a desensitizing toothpaste was not endorsed by the forum.

The peak prevalence of dentin hypersensitivity is in individuals aged between 30 and 40 years [4]. A problem 
of collecting prevalence data on dentin hypersensitivity is the supposed episodic nature of it and the validity of the measurements. After coverage of hypersensitive dentin, $30 \%$ of the persons still experience pain (West, personal communication at Gaba Forum 2011: Exposed cervical dentin and dentin hypersensitivity. November 2011, Basel CH), which suggests false-positive diagnoses in dentin hypersensitive patients.

The forum expected that the prevalence of dentin hypersensitivity will increase because more people will continue to have their own dentition into an older age probably increasing the number of sites of exposed dentin. The number of non-carious dentin lesions is expected to increase as a result of an increase of the erosiveness of the diet. In addition, erosiveness of the diet may prevent the closure of patent tubules. Intensified too zealous oral hygiene behavior may induce gingival recessions and prevent closure of patent dentinal tubules by removing fragile plugs. The forum emphasized, however, that there is no indication of the percentage of exposed dentin that will develop dentin hypersensitivity or whether there is a certain risk period after exposure. Therefore, the forum pleas for better and more epidemiological research on dentin hypersensitivity. These studies should also address risk factors and the reasons of the episodic nature.

\section{Toothbrush and toothpaste}

As far as the mechanism is involved, all members of the forum subscribe the hydrodynamic theory firstly suggested by Gysi [6] and later substantiated by Brännström [7]. Individual differences in hypersensitivity may primarily relate to the number and size of patent tubuli. The capability of reparative processes of the pulp/dentin complex and resulting tubule occlusion and the age of the pulp are likely to be very important factors in the susceptibility of an individual to experience the pain of dentin hypersensitivity [3]. Dentin may be exposed after gingival recession and loss of cementum or after the loss of enamel. The forum extensively discussed the role of toothbrush and toothpaste in this and recognized that the role of toothbrush and toothpaste as causing factor is frequently (especially by laymen) overestimated. The loss of cementum can hardly be prevented and there is a minimal risk of loss of enamel with twice a day normal brushing with modern toothbrushes, being hand or electric, and toothpastes with RDA values at or below the ISO standards (this is <200). In the loss of enamel, toothbrush and toothpaste are always co-factors and the main factor being erosion, abrasion, and attrition should always be diagnosed.

Dentin is softer than enamel and toothpaste and toothbrush may contribute to wear exposing deeper layers of dentin. Theoretically, this could aggravate dentin hypersensitivity as a result of increased ratio lumen area over surface area, but in the clinic, this relationship is not observed. In explanation, the tooth has reparative capability with reactionary and reparative dentin deposition reducing the patency of tubules [3]. To avoid dentine wear, low RDA toothpastes may be advised, although clinical evidence of the benefit is lacking.

The abrasiveness and chemical compounds of the toothpaste can, however, hinder the formation of plugs occluding patent tubules and remove a smear layer. The physical forces during the brushing exercise as such are not able to remove these [8]. But wrong brushing habits and too frequent brushing may cause gingival recession exposing root dentine. Several presenters had cited an epidemiological study in Dutch Adults showing that those who brushed three times daily had no less caries but more exposed root surfaces than those who brushed twice daily [9].On the other, hand no-brushers will develop more exposed root surfaces than brushers as a result of increased frequency of gingivitis and periodontitis. Parafunction habits do not correlate with the presence of exposed cervical dentin [3].

\section{Oral health-related quality of life}

Much attention was paid by the forum to the effect of dentin hypersensitivity on oral health-related quality of life $[8,10$, 11]. Very little research on this topic was identified but is it is clear that a substantial segment of persons suffering from dentin hypersensitivity seek treatment complaining of different types of discomfort such as pain while consuming hot or cold foods and beverages, during tooth brushing or sometimes even while breathing [10]. Using the OHIP G49 scale Bekes et al. [12] evaluated oral health-related quality of life in individuals seeking care for hypersensitive teeth. The mean OHIP summary score in these individuals was $34.5( \pm 22.6)$ while general population control subjects scored only $12.2( \pm 18.4)$ [8]. It is clear that this field of research has to be further explored.

The forum felt that there is also a need for a short list of questions to be used in the general practice for diagnosis and treatment evaluation. This questionnaire should preferably contain no more than five questions. Such a questionnaire can be derived from OHIP 14 although it was questioned whether a generic list would be appropriate or whether the questions should be specific to the individual and the conditions [11]. Such a questionnaire shifts the focus of clinicians from the oral cavity (tactile, cold, and air blast measurements) to the patient as a whole evaluating patient annoyance with dentin hypersensitivity. It would be of interest to compare the results of the two types of diagnosis and treatment evaluations (questionnaire versus 
stimulus-related evaluation) and when the results do not agree the question arises which evaluation is given priority. The forum noticed that the triggers to provoke dentin hypersensitivity in the dental office may be completely different from the triggers in patients' daily life. Moreover the triggers in the dental office are difficult to standardize.

\section{Ownership of the problem}

The forum agreed that the diagnosis of dentin hypersensitivity is a diagnosis of exclusion. It is important to acknowledge that there is a variety of conditions which may give the same sensations as dentin hypersensitivity that have all to be excluded before the diagnosis dentin hypersensitivity is justified $[1,8]$. It became evident that there is no commonly accepted methodology used in clinical practice for the evaluation of dentin hypersensitivity and its treatment. Apparently, a difference exists in perception between patients who self-report on the problem and the clinician's judgment when diagnosing and treating the condition $[8,13]$. This raises the question of ownership of the problem. Does the clinician have to look for dental hypersensitivity or only if the patients complain? Is the treatment successful when the patient is satisfied or should it objectively be established?

As dentin hypersensitivity is a diagnosis by exclusion, the individual cannot make the diagnosis. When a patient presents with presumed dentine hypersensitivity, the diagnosis has to be made by the dental professional. For the anamnesis the Socrates rule was presented by Gilliam: site, onset, character, radiation, associations, timing, exacerbating, and severity. The patient should be asked to identify pain inducing stimuli and the dental professional should ask for personal promoting habits and previous dental therapies [8]. It is suggested to use at least two different stimuli to provoke the pain and to repeat the stimuli when the site is covered for instance by a (fluoride) varnish.

\section{Treatment}

It is of course best to prevent exposed dentine surfaces. In this respect, it was suggested that there is a need for the description of accurate instrumentation for patients frequently visiting the dental clinic for scaling and rootplaning.

Once the diagnosis has been established, the clinician can suggest the various treatment options. It is important to recognize that both counseling and preventive aspects of the diagnostic and management of dentin hypersensitivity should not be forgotten [13]. For example, the use of diet history sheets to help the patient and clinicians to identify various erosive elements. Also, psychological aspects of dentin hypersensitivity when present should be addressed. First, advices are to avoid triggers and let nature (i.c. (re)mineralization from saliva and reactionary and reparative dentin deposition) do its job and close the patent tubules. Subsequently, the clinician can advise various products which are aimed to occlude patent tubules. For this, there are a number of products available. At least ten different products were identified for home care. The forum did not discuss the relative effectiveness of the products but recognized that in the light of the individual differences between patients products that are not effective for one maybe effective for the other. For this, a trial and error protocol maybe opportune. In the dental office minimal invasive therapies as fluoride, dentin bonding, sealants, and laser therapy and more invasive therapies like mucogingival surgery and pulpectomy are available. These possibilities are discussed in the presentation of Martens [1].

\section{Recommendations}

The forum advocated that more research has to be done in the field of hypersensitive dentin. The following outstanding issues for research were named:

- Good epidemiological estimations of the prevalence of dentin hypersensitivity

- Better understanding of the long term effects of periodontal treatment on dentine hypersensitivity

- Better understanding of risk factors and the episodic nature of the condition

- Better understanding of the risks and defense factors of gingival recession and dental erosion

- Better tools to measure oral health-related quality of life and reliable question(naire)s for in the dental office both for diagnosis as for treatment evaluation

- Development of a valid screening list with predisposing, initiating, and perpetuating risk factors

- Development of adequate diagnostic tools for the daily practice

Conflict of interest The author declares that he has no conflict of interest

\section{References}

1. Martens LC. A decision tree for the management of exposed cervical dentine (ECD) and dentine hypersensitivity. (this issue)

2. Addy M, Smith SR (2010) Dentin hypersensitivity: an overview on which to base tubule occlusion as a management concept. J Clin Dent 21:25-30

3. West NX, Lussi A, Seong J, Hellwig E. Dentine hypersensitivity: pain mechanisms and etiology of exposed cervical dentine. (this issue) 
4. Splieth C, Tachou A. Epidemiology of dentine hypersensitivity. (this issue)

5. Schmidlin PR. Current management of dentin hypersensitivity. (this issue)

6. Gysi A (1900) An attempt to explain the sensitiveness of dentin. $\mathrm{Br}$ J Dent Sci 43:865-868

7. Brännström M (1963) A hydrodynamic mechanism in the transmission of pain produced stimuli through the dentin. In: Anderson DJ (ed) Sensory mechanisms in dentin. Pergamon Press, Oxford, pp 73-79

8. Gernhardt CR. How valid and applicable are current diagnostic criteria and assessment methods for dentin hypersensitivity? An overview. (this issue)
9. Kalsbeek H, Poorterman JHG, Kivit MM. Tandheelkundige verzorging volwassen ziekenfondsverzekerden 1995-2002. Mondgezondheid, tandartsbezoek en preventief gedrag na de stelselherziening van 1995. Leiden: TNO Preventie en Gezondheid, 2003. Publ nr 03.219.

10. Bekes $\mathrm{K}$, Hirsch $\mathrm{C}$ What is known about the influence of dentine hypersensitivity on oral health-related quality of life. (this issue)

11. Sioux JL. How to make a link between oral health related quality of life and dentine hypersensitivity in the dental office. (this issue)

12. Bekes K, Schaller HG, Hirsch C (2008) Improvement of oralhealth related quality of life in subjects with dentin hypersensitivity. ZWR 117:136-142

13. Gilliam DG. Current diagnosis of dentine hypersensitivity in the dental office: an overview. (this issue) 